\title{
Itinéraires
}

Itinéraires Littérature, textes, cultures

\section{La pensée magique en Californie : Where I Was From de Joan Didion}

\section{Sophie Létourneau}

\section{(2) OpenEdition}

\section{Journals}

\section{Édition électronique}

URL : http://journals.openedition.org/itineraires/2674

DOI : $10.4000 /$ itineraires.2674

ISSN : 2427-920X

Éditeur

Pléiade

\section{Référence électronique}

Sophie Létourneau, "La pensée magique en Californie : Where I Was From de Joan Didion ", Itinéraires [En ligne], 2015-1 | 2015, mis en ligne le 18 décembre 2015, consulté le 21 avril 2019. URL : http:// journals.openedition.org/itineraires/2674; DOI : 10.4000/itineraires.2674

Ce document a été généré automatiquement le 21 avril 2019.

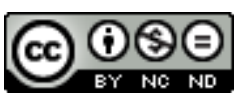

Itinéraires est mis à disposition selon les termes de la licence Creative Commons Attribution - Pas d'Utilisation Commerciale - Pas de Modification 4.0 International. 


\title{
La pensée magique en Californie : Where I Was From de Joan Didion
}

\author{
Sophie Létourneau
}

1 Aux États-Unis, Joan Didion se passe de présentation. Icône culturelle des années 1970, journaliste, romancière, scénariste, elle est surtout connue pour ses recueils d'essais Slouching Toward Bethleem (1968) et The White Album (1970), qui font partie du canon américain de la nonfiction. Par ses reportages sur la contre-culture (les hippies, les Doors, Joan Baez) et quelques grandes affaires criminelles (Charles Manson, Pattie Hearst, les Black Panthers), elle s'emploie à montrer que la réalité américaine est moins lisse qu'on ne voudrait le croire. Cette posture critique de même que la facture autobiographique de ses reportages rattachent Joan Didion au New Journalism. Aux codes de l'objectivité journalistique, jugée fallacieuse, les «nouveaux journalistes " préfèrent un reportage immersif, rendu par une narration à la première personne et une focalisation subjective. Truman Capote, Hunter S. Thompson, Norman Mailer, Gay Talese, Tom Woolf et, bien sûr, Joan Didion sont les principaux représentants de ce journalisme dit «littéraire », un genre où l'analyse politique n'exclut pas l'anecdote personnelle.

2 Citée par Bret Easton Ellis, Joyce Carol Oates et Zadie Smith parmi leurs influences marquantes pour son ton ironique, son style ciselé et son sens du détail parlant, Joan Didion n'a pourtant remporté un succès populaire et la consécration critique qu'en 2005 avec la publication du récit de deuil The Year of Magical Thinking. En plus d'un succès commercial, The Year of Magical Thinking a gagné le National Book Award et a fait l'objet d'une adaptation théâtrale sur Broadway. Ce livre portant sur le décès de son mari a été suivi, sept ans plus tard, par un récit portant sur la mort de leur fille: Blue Nights. (En France, L'Année de la pensée magique et Le Bleu de la nuit ont été publiés chez Grasset, en 2007 et en 2013.) Apolitiques, ces récits de deuil auraient toutefois le mérite de cantonner l'écriture autobiographique dans la sphère privée, selon certains commentateurs hostiles au mélange des genres caractéristique du New Journalism et de Joan Didion en particulier. Bruce Bawer, par exemple, dans un article publié par la Hudson Review, conseille à l'essayiste de s'en tenir au récit de deuil, un sujet mieux adapté à sa sensibilité narcissique 
que le 11-septembre ou la ville de Miami, sujets politiques sur lesquels Didion a écrit, qu'il aurait mieux valu laisser, selon lui, à des esprits moins solipsistes (Bawer 2007 : 87).

Or s'il est une chose que l'œuvre de Joan Didion ne cesse de questionner, c'est la séparation du personnel et du politique. Autant ses reportages que ses récits de deuil sont écrits depuis une posture autobiographique. Ce sont, à la lettre, des "récits de société ", c'est-à-dire des textes autobiographiques qui portent sur le monde dans lequel nous vivons. Des textes dans lesquels l'ego de l'écrivain se nourrit de l'ethos du journaliste. Cette intrication du personnel et du politique n'est jamais aussi probante que dans Where I Was From. Paru en 2003 aux États-Unis, le livre n'a pas encore été traduit en France. Il s'agit d'une enquête sur le rêve californien doublée d'un récit de deuil dans le but de cerner ce lieu rêvé qu'est la Californie - là où l'auteure est née en 1934 et là où sa mère, Eduene Jerret Didion, est décédée en 2001.

Dans cet article, j'examinerai la porosité de la frontière entre le personnel et le politique dans Where I Was From. Pour ce faire, je dégagerai d'abord les filons les plus importants de la réflexion de Didion. La structure de ses livres est en effet très complexe, pour ne pas dire touffue. L'argumentaire suit plusieurs fils narratifs, parfois abandonnés, pour être plus tard repris. Ce résumé me permettra de faire apparaître tant le propos de l'auteure que la manière dont elle s'y prend pour "démystifier " le rêve californien. Après avoir défini les trois mythes qui supportent le rêve californien selon Didion, j'étudierai les traitements narratif, esthétique et critique qu'elle leur fait subir. J'interrogerai ensuite la manière dont Didion rattache divers éléments du rêve californien aux souvenirs d'enfance. Pour Didion, le rêve californien est intimement lié aux croyances enfantines : il est porté par le devoir filial et se nourrit de nostalgie. En conclusion, je m'attacherai à la part la plus personnelle du livre, liée au deuil de la mère de l'auteure. Ce «récit de société » éminemment singulier se termine, en effet, avec celle qui «incarnait » les contradictions du Golden State et dont la mort marque la fin de toutes les illusions.

\section{Les trois mythes}

5 Tel qu'il est dépeint dans Where I Was From, le rêve californien repose sur trois mythes : celui de la vertu de ses habitants, celui de la fertilité de la terre californienne et celui d'une richesse perpétuelle.

6 Le premier mythe, celui de la vertu des habitants, s'appuie sur le mythe des origines. Après avoir tout abandonné au Kentucky ou au Tennessee, des pionniers ont traversé les plaines de l'Ouest en caravanes, risquant ainsi leur vie pour faire reculer la frontière américaine. On reconnaît le « grand récit » des origines de la Californie, sur lequel Didion se penche avec une pointe d'ironie dès le début de son essai. Selon elle, les articulations de ce récit évoquent la narration biblique. Les étapes connues de ce "pèlerinage » sont vénérées comme autant de "stations" au cours desquelles les pionniers sont mis à l'épreuve. Par exemple, un roc de granit sur la Piste de l'Oregon, «Independence Rock », doit son nom au fait qu'il fallait avoir traversé cette "station" avant la fête de l'Indépendance, au risque de se trouver devant une Sierra Nevada enneigée, impossible à traverser ${ }^{1}$. Édifiant, le récit de la traversée ( «crossing story») est aussi celui d'une rédemption. Beaucoup d'éléments rattachent ainsi le mythe des origines aux valeurs et aux récits protestants, faisant des pionniers des modèles de vertu, de valeur morale et d'endurance. De même, le paysage californien est décrit en des termes qui évoquent l'expérience mystique. 
7 Le second mythe qui participe du rêve californien a, lui aussi, une résonance biblique : il s'agit du mythe de la terre d'abondance. Le trope de la cornu copiae apparaît surtout dans les anecdotes liées à l'enfance de l'auteure. Mais la plus belle de ces anecdotes se trouve toutefois dans un article paru dans le recueil slouching Toward Bethleem (« Notes From a Native Daughter ») dans lequel Joan Didion se rappelle ses leçons de catéchisme. Une question est particulièrement parlante quant à la prégnance de cette croyance de la Californie comme éden maraîcher :

Q. In what way does the Holy Land resemble the Sacramento Valley? A. In the type and diversity of its agricultural products. ${ }^{2}$ (Didion $1968: 174$ )

Le dernier mythe abordé par Didion est celui d'une croissance perpétuelle. De la ruée vers l'or à la création d'une classe moyenne, le rêve californien est une variation sur le thème du rêve américain. "The American dream made affordable for a generation of industrial workers $^{3}$ ", écrit Donald J. Waldie, cité par Didion (2003: 106). Pour les parents et les enfants du babyboom, on a construit un réseau routier étendu et on a développé des lotissements en banlieue. La création de l'université de Californie et l'implantation des industries aérospatiale et technologique à Silicon Valley, ont participé de la promesse d'une prospérité sans fin.

\section{Une critique paradoxale}

Ce sont les récits et l'imagerie associés à ces mythes que Joan Didion déconstruit en mélangeant, d'abord, les genres et la provenance des textes qu'elle étudie.

Pour aborder le mythe des origines du peuplement de la Californie, par exemple, l'auteure analyse un discours prononcé en 1915 par Josiah Royce, professeur de philosophie à Harvard, qui voit dans la traversée une odyssée de signes et de tests (Didion 2003 : 32) et dans l'entreprise de colonisation, une forme de salut (Didion 2003: 28). En phase avec le discours protestant, ce discours contraste avec les extraits de journaux des pionniers, les photographies et les lettres de ses ascendants que Joan Didion cite, mais aussi avec le journal de la propre mère de Josiah Royce, Sarah, qui montrent la part sombre de cette traversée et l'ambiguité morale des pionniers. Dans la confrontation des récits et des points de vue, les détails contradictoires et les failles narratives (« narrative flaws ») apparaissent d'autant plus crûment. À l'idée de rédemption, tant valorisée dans le récit canonique, Joan Didion oppose une litanie d'abandons : adieux aux êtres chers au moment du départ, possessions dont on se défait en chemin, morts à qui l'on ne donne pas de sépulture - quand on ne les mange pas. On rappellera que le clan Donner-Reed est connu aux États-Unis pour avoir survécu à une traversée éprouvante (blizzards, faim, désorganisation, désorientation, manque d'équipement et décès de près de la moitié du groupe) en se livrant au cannibalisme. Les ancêtres de Joan Didion faisaient partie du convoi, mais s'en sont séparés avant que n'ait lieu cet épisode qui renverse le mythe de la terre promise, comme le souligne H. Jennifer Brady :

the experience of these families has become the original antimyth of the golden

land in which the promised land becomes the heart of darkness. (Brady 1979 : 455)

11 Joan Didion se réfère souvent à cette équipée tragique afin de souligner l'envers noir de la vertu des pionniers. Au récit canonique d'une odyssée vers l'Ouest, elle oppose celui d'une « lutte mesquine pour la survie » - « a mean scrambling for survival » (Didion $2003: 35$ ) -, telle que l'histoire de Miss Gilmore en offre une autre illustration. Un émigrant nommé Bernard J. Reid rapporte avoir rencontré une jeune fille de dix-sept ans (Miss Gilmore), 
que les gens de son convoi avaient abandonnée à son frère, atteint du choléra. Par crainte d'être ralentis, puis surpris par l'hiver, ces pionniers les ont laissés derrière eux. Comme souvent pour marquer un paradoxe (ici, celui de la valeur rédemptrice du voyage), Joan Didion pose une question, dont le ton moralisateur n'est pas sans détourner le discours religieux qui a inspiré le mythe de la vertu des premiers colons :

When you jettison others so as not to be 'caught by winter in the Sierra Nevada mountains', do you deserve not to be caught?5 (Didion 2003 : 37)

\section{Démonter le mythe par le montage}

12 La critique de Didion repose pour beaucoup sur un effet de montage. En faisant s'entrechoquer plusieurs discours, elle met de l'avant le paradoxe que cache le mythe. Le même procédé est employé pour démonter le mythe de la fertilité de la Californie, terre sainte (« Holy Land»), et le mythe de la richesse de ses habitants. Aux discours officiels du gouvernement américain et aux Mémoires plein d'enthousiasme d'anciens propriétaires terriens, Joan Didion oppose des lithographies de Sacramento submergé par les inondations annuelles et des statistiques liées à l'agriculture et à l'irrigation, mettant ainsi à mal non seulement le mythe d'une terre d'abondance, mais également celui de l'esprit entrepreneurial des Californiens. Il apparait, en effet, que l'agriculture californienne est dépendante du gouvernement fédéral pour le financement d'un environnement artificiel, sans qu'elle ne réponde aux besoins du marché.

Four hundred thousands acres were planted in rice, the cultivation of which involves submerging the fields under six inches of water from mid-April until the August harvest, months during which, in California, no rain falls. [...] Ninety percent of this California rice was glutinous medium-grain Japonica, a type not popular in the United States but favored in both Japan and Korea, each of which banned the import of California rice. ${ }^{6}$ (Didion $2003: 25-26$ )

13 À la réalité d'une terre cultivée de manière industrielle, l'idée d'un éden californien apparaît comme un mirage qui trouve sa représentation dans les tableaux de Thomas Kinkade, peintre populaire de paysages bucoliques empreints d'une aura lumineuse (le « Kinkade Glow») ainsi que chez les impressionnistes de la collection du musée Irvine, dont les tableaux rustiques vides de toute présence humaine (sauf peut-être un Indien en canot) oblitèrent le développement et l'étalement urbain auxquels la famille Irvine a ellemême contribué.

$\mathrm{Au}$ final, c'est peut-être moins la réalité du rêve californien que Joan Didion met en doute en critiquant les mythes qui le composent, que sa viabilité à long terme et son actualité. Depuis ses débuts, la Californie voit sa population augmenter de moitié à chaque décennie (Didion 2003: 172-173). Aimantés par le rêve d'une nouvelle vie à construire, les nouveaux arrivants carburent eux aussi au fantasme d'un perpétuel boom économique. Si l'on ajoute la récente délocalisation de l'industrie aérospatiale, la montée du chômage et de la délinquance juvénile, on peut penser que le rêve californien s'est transformé en cauchemar. À l'aide d'une véritable mosaïque de récits d'explication, incluant un fait divers (l'affaire «Spur Posse », une fraternité d'adolescents accusés de viols multiples), l'analyse de sa couverture médiatique, un vox populi et un portrait socio-économique de Lakewood, ville de banlieue où les délits ont été commis, Joan Didion ouvre le mythe à son envers paradoxal : le marasme économique dans lequel l'État est plongé depuis les années 1990, une réalité que ses résidants préfèrent toutefois nier. 


\section{Désenchanter le mythe}

15 Tout au long de l'essai, le ton employé par Didion donne à penser que, à défaut des faits, la nature paradoxale du rêve californien est connue du lecteur et des Californiens euxmêmes. Bien qu'elle identifie chacun des mythes, puis qu'elle juxtapose les images d'une Californie idéalisée et celles d'une Californie réelle, Joan Didion ne donne pas dans le journalisme d'enquête, dont la visée serait de révéler une vérité «cachée ». Toutes les contradictions relevées servent moins à déboulonner les mythes qu'elles ne participent d'une plus grande contradiction, d'un plus grand paradoxe, à savoir : la croyance délibérée au rêve californien en dépit du bon sens.

16 Where I Was From s'articule autour de l'idée que la Californie est une illusion. Une promesse à laquelle sa famille a voulu croire. An enchantment, écrit Didion, auquel elle aurait elle-même succombé. "La pensée magique en Californie »: tel aurait pu être le sous-titre à saveur ethnographique du livre. "It's not so much about California as it is about a state of mind, an enchantment. That's where I was from ${ }^{7}$ ", confiait Joan Didion à un journaliste à la sortie du livre (O'Herir 2003). Bien qu'il soit soutenu par des décisions politiques, le rêve californien est porté par ses «croyants ». Aussi est-ce le credo plutôt que la doxa, la croyance au mythe plutôt que le processus de mystification, qui intéressent Didion. En racontant comment ses ancêtres, ses parents et elle-même ont intériorisé le credo californien ("We believe in fresh starts. We believe in good luck ${ }^{8}$ ", Didion 2003 : 128), Joan Didion ne se contente pas de décrire le rêve californien: elle en montre l'emprise. Par le relais de la mémoire familiale, de conversations et de scènes domestiques arrachées au passé, la méthode de Didion se fait proprement littéraire. Le mythe n'est plus un objet d'étude ou une grille d'analyse, mais le prisme à travers lequel l'individu s'explique sa propre histoire et décide de son destin.

17 "We tell ourselves stories in order to live ${ }^{9}$ ", disait Joan Didion au début de son livre culte, The White Album (Didion 1979 : 11). De la puissance narrative du mythe, Joan Didion fait un enjeu existentiel. Pour le jauger, elle privilégie la forme de l'anecdote. Pourquoi l'anecdote? Récit d'un incident qui s'est réellement produit, l'anecdote est fondée sur un détail emblématique. C'est par ce détail que l'anecdote résiste au récit de société. Il fait tache en incarnant un paradoxe qu'il met en évidence.

18 Au début de Where I Was From, une Joan Didion âgée d'environ quatorze ans porte un collier de cristal que lui a prêté sa mère pour combattre les effets de la canicule. C'est sa graduation de la middleschool et elle lit un discours (qu'elle a écrit) intitulé : "Our California Heritage» («La Californie pour patrimoine»). L'assurance que lui donne le collier n'a d'égal que l'attitude confiante qui teinte sa vision de l'histoire de la Californie, objet de son allocution. Didion essayiste se sert de ce texte pour illustrer le mythe des origines. Mais ce que l'anecdote éclaire surtout, c'est la pensée magique au cœur du rêve californien, pensée magique dont est investi le collier de cristal.

Tel qu'il est décrit par Didion, le rêve californien s'apparente à une croyance enfantine de toute-puissance. Le caractère infantile de cette croyance apparaît clairement dans la comparaison des mots employés sur le site Web du US Bureau of Reclamations et dans "Our California Heritage ", l'allocution que Didion a écrite vers l'âge de treize ans. Le US Bureau of Reclamations écrit qu'il a suffi d'un peu d'eau pour faire de cette vallée stérile le potager de la nation ( just by adding water, this vale of sterility would bloom as the nation's garden », Didion 2003 : 48). Un même enthousiasme caractérise la vision de la 
petite Joan dans "Our California Heritage »: "We had an irrigation problem, so we built the greatest dams the world has known ${ }^{10} »$ (Didion 2003 : 49). Et c'est toute la naïveté du rêve californien qui apparaît dans la confrontation des deux énoncés, une naïveté dont Didion mettra des années à se départir (Didion 2003 : 17). Pourquoi tant d'années ? Selon elle, les Californiens vivent dans un commun déni, profondément persuadés qu'ils sont de leur innocence (Didion 2003 : 71).

\section{La perte de l'innocence} C'est ainsi que le processus de démystification de l'argumentaire se superpose d'un récit tantôt mélancolique, tantôt ironique, de la perte de l'innocence.Cette prise de conscience politique ne vient pas sans une certaine amertume, que Joan Didion évoque dans les termes d'un désenchantement. Plutôt que d'être sous le coup d'une révélation, l'individu fait l'épreuve de sa résistance, car on ne peut se défaire de l'emprise du mythe sans renoncer à la satisfaction enfantine que donne la pensée magique. Pas plus qu'on ne peut résister aux « récits de société » sans renier ceux qui nous les ont transmis. Pour Joan Didion, ces récits sont des " histoires qu'on m'a racontées toute ma vie », « des fragments d'histoire orale locale préservés par les filles et les petites-filles sur des tablettes de papier et à l'endos d'enveloppes » (Didion 2003 : 157). emprise. Pour étudier ce phénomène, Joan Didion se penche sur sa propre nostalgie, telle que son premier roman, Run Rivers (1963), l'illustre. Selon l'analyse thématique qu'elle fait de ce roman situé dans la vallée de Sacramento, la transformation de la Californie est présentée comme un déclin :

It was true that the habits and customs of "Old Sacramento" (the school vacation jobs on the ranches and at the canneries, the swimming in the rivers and wading in the ditches, the dutiful study of agricultural exhibits at the California State Fair) were giving way to a more urban, or suburban, life, in which children swam in clear water in backyard pools lined with gunite and bought Italian typewriters and ate pears bought in supermarkets rather than dropped off in lugs by the relatives who grew them..$^{12}$ (Didion $2003: 166$ )

Si Didion concède que Sacramento a changé au moment de la rédaction de Run River et pendant les quarante ans qui sépare cette publication de celle de Where I Was From, en revanche, elle se montre sceptique devant sa propre nostalgie. D'une part, elle constate que la seule constante en Californie, ce sont les «booms ", les changements (Didion 2003 : 167). D'autre part, elle est d'avis que la nostalgie relève d'un certain élitisme. Regretter la Californie des débuts, c'est regretter un territoire peuplé de quelques grands 
propriétaires terriens. La majorité des gens qui habitent la Californie aujourd'hui n'auraient pas eu les moyens de s'y installer au temps des premiers colons (Didion 2003 : 101). Aussi Joan Didion se sert-elle de sa nostalgie devant la transformation du paysage californien pour questionner un quatrième mythe au fondement du rêve californien: celui d'un paradis perdu.

Enfant, Joan Didion entendait répéter que l'herbe poussait aussi haut que le dos des chevaux quand la Californie était encore vierge de tout développement urbain. Aujourd'hui, elle soutient que ce mythe du paradis perdu entretient l'exclusion des nouveaux arrivants. Pour illustration, Joan Didion raconte que son grand-père a un jour écrit une lettre aux éditeurs d'un manuel scolaire, jugeant que l'histoire de la Californie avait été réécrite (« history rewritten») afin de plaire aux nouveaux arrivants («new people ») (Didion 2003 : 96). Comme le mythe des origines renforce la cohésion du groupe, le mythe du paradis perdu justifie l'hostilité des Californiens devant les «new people». Ce sont pourtant les anciens Californiens, écrit Didion, qui ont spolié les terres vierges de la Californie en s'y installant pour ensuite s'enrichir aux dépens des nouveaux en leur vendant des lots. La nostalgie des Californiens ne serait que le vernis acceptable de la conviction qu'ils ont d'être dans leur droit ("the conviction of entitlement») (Didion 2003: 79). C'est toute l'ambiguïté de ce sentiment de propriété qui transparaît dans la décision qu'a prise la famille de Didion de mettre en vente le cimetière où leurs ancêtres ont été enterrés.

Chez Didion, le caractère problématique du mythe du paradis perdu, l'ambivalence que suscitent la nostalgie de l'enfance et le récit de la perte de l'innocence, posent la question du legs et de la filiation. Dans la mesure où le mythe est transmis, peut-on dire qu'il s'agit d'un héritage ? D'un "patrimoine ", pour reprendre le titre de l'allocution de la jeune Didion. Si l'on refuse le mythe, renie-t-on ses parents? Doit-on faire le deuil du mythe comme on en vient un jour à faire le deuil de ses parents?

\section{Mythe et filiation}

Après avoir identifié les mythes au cœur du rêve californien et montré que la «pensée magique " s'ancre dans l'enfance, Joan Didion conclut sa réflexion par un récit de deuil. La dernière partie de Where I Was From est en effet consacrée à la mère de l'auteure, Eduene Jerret Didion, dont la mort a précédé la rédaction du livre. Or ce récit de deuil ne se limite pas à la sphère familiale, intime. Comme toujours chez Didion, le regard autobiographique a portée politique. Car si Didion conclut son essai sur la Californie par un récit de deuil, c'est que sa mère portait les contradictions de la Californie (Didion 2003 : 204). Ce n'est qu'en revenant sur la vie de cette Californienne typique que l'auteur parvient à faire le deuil du rêve californien, de la croyance aux mythes et de la pensée magique. Nulle part mieux que dans cette coda on ne voit se tresser le personnel, le politique et le narratif en un « récit de société » éminemment singulier.

Dans ce récit de deuil, il est question, bien sûr, de départs et d'abandons : la mort de la mère incite la fille à se rappeler les séparations qui ont marqué leur relation en même temps qu'elle n'est pas sans évoquer les fantômes du clan Donner-Reed et celui de Miss Gilmore. Il est question également de l'enfant qu'a été Didion et dont personne ne se souviendra désormais. Disparue le dernier témoin de son enfance, mais l'un des derniers témoins aussi de la « Vieille Californie ». De tout le livre, l'anecdote la plus mémorable est 
assurément celle où Joan Didion raconte avoir conduit sa mère sur la route qui va de Monterey à Berkeley, la même qu'elles avaient l'habitude d'emprunter avant le décès du père de Didion. Or sa mère refuse de croire qu'elles sont sur le bon chemin. Sa fille la rassure, lui répète qu'elles sont bien sur la route 101. Alors la mère demande : «Then where did it all go ?» (« Mais où tout est parti ?»)

She meant where had San Benito and Santa Clara Counties gone as she remembered them, the coastal hills north of Salinas, the cattle grazing, the familiar open vista that had been relentlessly replaced (during the year, two years, three, the blink of the eye during which she had been caring for my father) by mile after mile of pastel subdivisions and labyrinthine exists and entrances to freeways that had not previously existed. ${ }^{13}$ (Didion $2003: 215-216$ )

Mise en abîme de Where I Was From, l'anecdote dit une certaine nostalgie et la fin des illusions. Elle évoque également l'amertume qui anime Joan Didion lorsqu'elle fait le récit de ses désenchantements. Et dans la mesure où la mère, en deuil du père, doit faire le deuil d'un passé dont le décor a disparu, l'anecdote parle finalement du propre deuil de Didion, du double deuil de la mère et de l'idée qu'elle se faisait de la Californie dont Where I Was From se veut le récit.

Si la mélancolie de la mère n'offre pas de solution aux paradoxes soulevés dans l'essai, la fin du livre présente une scène s'approchant de l'épiphanie. Devant la joie de Quintana, la fille adoptive de Didion, qui s'amuse sur les trottoirs en bois d'une reconstitution du Vieux Sacramento, se produit l'ultime " prise de conscience », du livre et la fin de toutes les illusions. Sur le point de dire à sa fille adoptive que sa famille se baladait, jadis, dans le Vieux Sacramento, Joan Didion se rend compte, d'abord, que sa fille n'entretient pas de lien de sang à ses ancêtres, et, ensuite, qu'elle-même n'est pas plus réellement attachée à cette reconstitution que sa fille adoptive (Didion 2003 : 219). La reconstitution comme le mythe n'a qu'un «effet décoratif » et l'hérédité ne nous attache qu'imaginairement au passé : tel est la fin «heureuse » de Where I Was From. Au terme d'une longue critique du rêve californien, suivi du récit du deuil de sa mère, Joan Didion conclut de manière ambiguë. Le mythe n'est pas réel, le rêve californien non plus. Ce ne sont que des histoires que nous nous racontons. Mais qu'importe, écrit Didion, puisqu'il n'y a pas de vraie manière, puisqu'on ne peut pas réellement faire le deuil de ce qui nous échappe : «There is no real way to deal with everything we loose » (Didion $2003: 225$ ).

\section{Conclusion}

30 À la lecture du récit qui conclut le livre, on comprend que toute cette entreprise de « démystification » est, en fait, un récit de deuil politique : deuil du rêve californien et de ses mythes dorés, mais deuil aussi d'une certaine innocence qu'il n'est plus possible de conserver. Chez Didion, l'écriture autobiographique ne s'«oppose» pas à l'analyse politique, pas plus qu'elle ne s'y « ajoute » ou qu'elle ne le "parasite». Dans Where I Was From, le récit de deuil donne véritablement forme à l'analyse politique. Et c'est sans doute l'aspect le plus singulier de ce livre que de faire du genre éminemment intime qu'est le récit de deuil l'archétype du "récit de société ». 


\section{BIBLIOGRAPHIE}

Bawer, Bruce, 2007, « Didion's Dreamwork », The Hudson Review, vol. 60, n 1, p. 85-103.

Brady, H. Jennifer, 1979, « Then and Now: The Fiction of Joan Didion », Contemporary Literature, vol. $20, \mathrm{n}^{\circ} 4$, p. $452-470$.

Didion, Joan, 1968, Slouching Toward Bethleem, Farrar, New York, Straus and Giroux.

,- 1979, The White Allbum, New York, Farar, Straus and Giroux.

-, 2003, Where I Was From, New York, Random House.

O’Hehir, Andrew, 2013, « Golden State of Hipocrisy », Salon.com [Site web], http:// salon.com/2003/10/18/didion_4/, consulté le 3 juin 2013.

Scatamacchia, Cristina, 2004, « Horizontal and Vertical Themes in Joan Didion's Memoir Where I Was From », Rivista di studi anglo-americani, $\mathrm{n}^{\circ}$ 15-16, p. 69-91.

\section{NOTES}

1. «Independence Rock [...] was so named because the traveler who had not reached that point by the Fourth of July, Independence Day, would not reach the Sierra Nevada before snow closed the passes » (Didion $2003: 33$ ).

2. «Question: De quelle manière la Terre Sainte ressemble-t-elle à la vallée de Sacramento ? Réponse : Dans la nature et la diversité de sa production agricole. » Les traductions en français de cet article sont les miennes.

3. «Une version abordable du rêve américain pour toute une génération d'ouvriers ».

4. «[C]e que ces familles ont vécu est devenu l'antimythe originel de la Californie : la terre promise se transforme en cœur des ténèbres. »

5. «Lorsque vous abandonnez vos prochains pour ne pas être "pris par l'hiver dans les montagnes de la Sierra Nevada", méritez-vous de ne pas être pris?»

6. "Quatre cent mille acres [environ $1600 \mathrm{~km}^{2}$ ] sont consacrés au riz, une culture qui exige que les champs soient inondés sous six pouces d'eau [environ $15 \mathrm{~cm}$ ] de la mi-avril aux récoltes d'août, une période pendant laquelle, en Californie, la pluie ne tombe pas. [...] Quatre-vingt-dix pourcent de ce riz californien est de variété japonica, un riz à grain moyen, glutineux, qui n'est pas en vogue aux États-Unis, mais qui est apprécié au Japon et en Corée, qui en ont interdit l'importation. »

7. «Le sujet n'est pas tant la Californie qu'un état d'esprit, un ensorcellement. Voilà d'où je viens. »

8. « Nous croyons aux nouveaux départs. Nous croyons à la chance. »

9. « Nous nous racontons des histoires pour vivre".

10. «Nous avions un problème d'irrigation, alors nous avons bâti les plus grandes digues que le monde ait connues $»$.

11. «Her work succeeds in illuminating that these core beliefs are only myths not coinciding with reality, and yet they have always mattered to the politics of California, and they still influence the life and expectations of its inhabitants » (Scatamacchia $2004: 85$ ). 
12. "Il est vrai que les us et coutumes du "Vieux Sacramento" (les emplois d'été dans les ranchs et les conserveries, la baignade dans les rivières et patauger dans les fossés, l'étude respectueuse de l'exposition agricole à la California State Fair) ont fait place à une vie plus urbaine (ou banlieusarde) dans laquelle les enfants nagent dans l'eau claire de piscines résidentielles bordées de béton et achètent des machines à écrire italiennes et mangent des poires achetées au supermarché plutôt qu'apportées par les membres de la parenté qui les cultivaient. »

13. «Elle voulait dire où les comtés de San Benito et de Santa Clara tels qu'elle se les rappelait sont partis, les collines sur la côte du nord de Salinas, les troupeaux qui paissent, l'horizon ouvert qui lui était familier et qui avait été infatigablement remplacé (durant l'année, les deux années, trois, le battement pendant lequel elle a pris soin de mon père) par des miles et des miles de subdivisions pastel et de sorties et d'entrées labyrinthiques menant à des autoroutes qui n'existaient pas avant. »

\section{RÉSUMÉS}

Écrivaine américaine associée au New Journalism, Joan Didion s'est intéressée au rêve californien. Dans un essai intitulé Where I Was From, elle questionne l'emprise de certains «mythes » sur la pensée politique des Californiens. Plutôt que de restreindre son champ d'investigation au domaine public, Joan Didion étudie également l'histoire de sa famille. Cette démarche, qui se situe entre le journalisme et le récit autobiographique, s'appuie sur une documentation qui relève aussi bien de l'histoire de la Californie (récits historiques, rapports gouvernementaux, faits divers, textes littéraires, tableaux) que de sa propre vie (archives familiales, souvenirs de jeunesse). Ce travail de recherche et de réminiscence, qui vise à démystifier la Californie, se conclut par un travail de deuil, qui marque la fin des illusions. Entre journalisme littéraire et récit de deuil, Where I Was From questionne le « récit de société » dans un parcours singulier que cet article s'emploie à retracer.

American writer Joan Didion, one of the most proeminent New Journalist, made the Californian dream the main topic of Where I Was From. In this essay, she criticizes the influence of "myths" on the political thought of Californians. Rather than restricting her field of investigation to the public sphere, Joan Didion also examines the story of her family. To convey her research, she borrows both to journalism and memoirs writing: her documentation is based on historical data, government reports, news in brief, literature and arts, but she also draws on family archives, oral history and childhood memories, offering a unique "récit de société" in which politics crosses autobiography. This article shows how Where I Was From aims to demystify the Californian dream through research, reminiscence, and the work of mourning by which the author finaly experiences the end of the "enchantment."

\section{INDEX}

Mots-clés : journalisme littéraire, récit de deuil, Californie, Joan Didion, Where I Was From Keywords : new journalism, memoirs of Loss, California, Joan Didion, Where I Was From 


\section{AUTEUR}

\section{SOPHIE LÉTOURNEAU}

Université Laval 